\title{
Entanglement, fidelity, and quantum-classical correlations with an atom walking in a quantized cavity field
}

\author{
S.V. Prants, M.Yu. Uleysky, and V.Yu. Argonov \\ Laboratory of Nonlinear Dynamical Systems, \\ V.I. Il'ichev Pacific Oceanological Institute of the Russian Academy of Sciences, \\ 690041 Vladivostok, Russia
}

(Dated: April 10, 2018)

\begin{abstract}
Stability and instability of quantum evolution are studied in the interaction between a two-level atom with photon recoil and a quantized field mode in an ideal cavity, the basic model of cavity quantum electrodynamics (QED). It is shown that the Jaynes-Cummings dynamics can be unstable in the regime of chaotic walking of the atomic center-of-mass in the quantized field of a standing wave in the absence of any kind of interaction with environment. This kind of quantum instability manifests itself in strong variations of reduced quantum purity and entropy, correlating with the respective classical Lyapunov exponent, and in exponential sensitivity of fidelity of quantum states to small variations in the atom-field detuning. The connection between quantum entanglement and fidelity and the center-of-mass motion is clarified analytically and numerically for a few regimes of that motion. The results are illustrated with two specific initial field states: the Fock and coherent ones. Numerical experiments demonstrate various manifestations of the quantum-classical correspondence, including dynamical chaos and fractals, which can be, in principle, observed in real experiments with atoms and photons in high finesse cavities.

PACS numbers: 42.50.Vk, 05.45.Mt, 05.45.Xt
\end{abstract}

\section{INTRODUCTION}

Entanglement is a birth-mark of quantum mechanics. A pure state of a closed quantum system evolves as a pure state, and all the measures of entanglement remain constant during the evolution. However, if the system is composed of interacting subsystems, the reduced density matrix of one of them - a measure of coherence lost in this subsystem - does not evolve in a unitary way. That is the reason of the loss of coherence in a system interacting with an environment composed of a large number of degrees of freedom. A lot of work has been done to understanding decoherence, dynamics of entanglement, and quantum chaos. One of the themes is the behavior of entanglement in bipartite quantum systems in the parameter regimes where their underlying classical analogues are chaotic in the sense of exponential sensitivity to small changes in initial conditions.

The basic models of quantum optics and cavity quantum electrodynamics are the Jaynes-Cummings model [1], describing the interaction between a two-level atom at rest and a quantized mode of the radiation field, and the Dicke model [2], describing the interaction of a collection of two-level atoms, located within a distance much smaller than the radiation wavelength, with a quantized mode of the radiation field. It is known for a long time 3, 4] that the classical limit of the Dicke model can be chaotic only beyond the rotating-wave approximation. It has been found that initial increase in the reduced linear entropy is faster for the initial states, prepared as wave packets centered at chaotic regions of the classical phase space, as compared to regular ones [5, 6]. The similar property has been found in the model of weakly-coupled quantum tops [7] and in the model of two-component
Bose-Einstein condensates [8].

In the above cited and other similar works, closed quantum systems have been considered, and the connection has been studied between quantum entanglement and dynamical chaos in the classical version of a quantum system under consideration, i. e. between two different systems. However, the classical limit of a quantum system is not unique. For example, there are different classical limits of the Jaynes-Cummings model [1] and its generalization, the Dicke model 2], which even have different names: neoclassical, quasiclassical, and semiclassical [9, 10]. One may treat atoms quantum mechanically and the field classically. There is a choice how to couple the atoms with their radiation field. The classical limit can be taken for both the atomic and field subsystems. As a result, one derives different equations of motion for expectation values of quantum operators for the same physical system whose phase spaces may have different properties. It is a problem to decide which classical limit is "the best one" and should be used for comparison with quantum treatment of the system.

In this paper we study the connection between the purely quantum characteristics, such as entanglement and fidelity, and classical chaos in the same system with dynamically coupled quantum and classical degrees of freedom. The quantum dynamics of a system with classical degrees of freedom is of great interest not only from the point of view of quantum chaos but from the view point of the correspondence between quantum and classical mechanics as well.

The main purpose of this paper is to show that entanglement between the atomic and field quantum states correlates with the center-of-mass motion of an atom in the standing-wave cavity field. Regular center-of-mass 
motion corresponds to a regular evolution of the reduced quantum entropy and fidelity of quantum states, whereas quantum evolution is unstable under a chaotic (random) walking of the atom. Quantum instability may arise in quantum-classical hybrid without introducing a bath with infinitely many degrees of freedom or any kind of noise to model environment whose role is played in our model by a translational degree of freedom. We prove that correlations between purely quantum characteristics, such as entanglement and fidelity of the atom-field states, and the classical measure of motion - the maximal Lyapunov exponent - arise in a natural way with a two-level atom moving in a single-mode quantized cavity field. By numerical simulation we demonstrate various manifestations of the quantum-classical correspondence, including dynamical chaos and dynamical fractals, that may be, in principle, found in real experiments with atoms and photons in high finesse cavities.

Atoms, interacting with the electromagnetic field, change both their internal electronic states and external translational states, the process known as a photon recoil. In the strong-coupling regime, the respective Hamiltonian describes the interaction between the field, electronic, and translational degrees of freedom. In our previous papers [11, 12, 13] we have studied the Hamiltonian dynamics of a two-level atom in a self-consistent standing-wave cavity field in the semiclassical approximation in the limit of an infinite number of photons and found both regular and chaotic regimes of motion of the atomic center of mass. The chaotic walking of atoms takes place in a stationary standing wave and is quantified by the positive values of the maximal Lyapunov exponent 11]. Typical chaotic atomic trajectories consist of intervals of random walking interrupted by regular oscillations near the bottom of some wells of the optical potential and long ballistic flights with practically constant velocity (so-called Lévy flights 14]). In the language of dynamical system's theory, there is a fractallike chaotic invariant set consisting of all chaotic and unstable periodic orbits in the phase space of the strongly coupled atom-field system. As a sequence, a scattering of atoms even at a $1 \mathrm{D}$ standing electromagnetic wave is fractal with prominent self-similarity of their scattering function, for example, in dependence of exit time on the atomic initial momentum [13].

Classical instability is usually defined as an exponential separation of two nearly trajectories in time with an asymptotic rate given by the maximal Lyapunov exponent $\lambda$. Perfectly isolated quantum systems are unitary and cannot be unstable in this sense even if their classical limits are chaotic 15]. It has been long ago proposed [16] to measure quantum instability by the decay of fidelity or overlap of two initially identical wave functions that evolve under slightly different Hamiltonians. In a number of numerical studies (see, for example Refs. 17, 18, 19, 20, 21] and cited therein) for a variety of classically chaotic models, it has been found that the overlap decay is, in general, not universal and de- pends on the strength of perturbations in Hamiltonians and other factors determining which decay regime prevails, algebraic, Gaussian, or exponential one. The usual strategy is to compare the fidelity decay to a separation of nearby trajectories in the phase space of a classical analogue of the quantum system under consideration. With our dynamically-coupled quantum-classical hybrid, a correlation between quantum and classical measures of instability arises in a natural way as a result of coupling between different characteristics of the same system.

The paper is organized as follows. In Sec. II we derive the Hamilton-Schrödinger nonlinear equations, describing interaction between a two-level atom with the classical translational degree of freedom and a single-mode quantized field in an ideal cavity, and discuss possible regimes of the center-of-mass motion in dependence on the values of the detuning of the atom-field resonance. We express linear and von Neumann entropies (measures of the atom-field entanglement) and fidelity (measure of instability of the Jaynes-Cummings quantum evolution) in terms of the respective probability amplitudes. In some cases we find exact and approximate solutions of the equations of motion. In Sec. IIII we study correlations between the entanglement and fidelity and the mode of the center-of-mass motion in a Fock-state cavity field. In this case the infinite-dimensional set of the equations of motion reduces to a few coupled nonlinear equations which are analyzed for different regimes of the center-of-mass motion. In fact, we deal with entanglement between two similar quantum systems, a two-level atom and a three- (or two-) level field. We find exact and approximate solutions for the entanglement in the Fock field and demonstrate numerically the close correspondence between these quantum characteristics and underlying classical chaos. In Sec. IV we report on correlations found between the entanglement and fidelity and classical random walking of an atom in a coherent-state field which are quantified in terms of the respective classical maximal Lyapunov exponent. Section $\nabla$ is for concluding remarks.

\section{A TWO-LEVEL ATOM WALKING IN A QUANTIZED CAVITY FIELD}

\section{A. Hamilton-Schrödinger equations of motion}

To specify the problem we consider the standard model in cavity QED with the Jaynes-Cummings Hamiltonian [1]

$$
\begin{aligned}
\hat{H}=\frac{\hat{p}^{2}}{2 m_{a}}+\frac{1}{2} \hbar \omega_{a} \hat{\sigma}_{z} & +\hbar \omega_{f}\left(\hat{a}^{\dagger} \hat{a}+\frac{1}{2}\right)- \\
& -\hbar \Omega_{0}\left(\hat{a}^{\dagger} \hat{\sigma}{ }_{-}+\hat{a} \hat{\sigma}_{+}\right) \cos k_{f} \hat{x}
\end{aligned}
$$

which describes the interaction between a two-level atom (with lower $|1\rangle$ and upper $|2\rangle$ states, the transition frequency $\omega_{a}$, and the Pauli operators $\hat{\sigma}_{ \pm, z}$ ) and a quan- 
tized electromagnetic-field mode (with creation $\hat{a}^{\dagger}$ and annihilation $\hat{a}$ operators) forming a standing wave with the frequency $\omega_{f}$ and the wave vector $k_{f}$ in an ideal cavity. The atom and field become dynamically entangled by their interaction with the state of the combined system after the interaction time $t$

$$
|\Psi(t)\rangle=\sum_{n=0}^{\infty}\left(a_{n}(t)|2, n\rangle+b_{n}(t)|1, n\rangle\right)
$$

to be expanded over the Fock field states $|n\rangle, n=$ $0,1, \ldots$ Here $a_{n}(t)$ and $b_{n}(t)$ are the complex-valued probability amplitudes to find the field in the state $|n\rangle$ and the atom in the states $|2\rangle$ and $|1\rangle$, respectively. In the process of emitting and absorbing photons, atoms not only change their internal electronic states but their external translational states change as well due to the photon recoil effect. If atoms are not too cold and their average momenta are large as compared to the photon momentum $\hbar k_{f}$, one can describe the translational degree of freedom classically.

The whole dynamics is now governed by the HamiltonSchrödinger equations 22] that have the following normalized form in the frame rotating with the frequency $\omega_{f}(n+1 / 2)$ :

$$
\begin{gathered}
\dot{x}=\omega_{r} p, \\
\dot{p}=-2 \sin x \sum_{n=0}^{\infty} \sqrt{n+1} \operatorname{Re}\left(a_{n} b_{n+1}^{*}\right), \\
i \dot{a}_{n}=-\frac{\delta}{2} a_{n}-\sqrt{n+1} b_{n+1} \cos x \\
i \dot{b}_{n+1}=\frac{\delta}{2} b_{n+1}-\sqrt{n+1} a_{n} \cos x
\end{gathered}
$$

with simple solution for the probability amplitude $b_{0}(\tau)$

$$
b_{0}(\tau)=b_{0}(0) e^{-i \delta \tau / 2} .
$$

Here $x \equiv k_{f}\langle\hat{x}\rangle$ and $p \equiv\langle\hat{p}\rangle / \hbar k_{f}$ are the atomic centerof-mass position and momentum, respectively. Dot denotes differentiation with respect to dimensionless time $\tau \equiv \Omega_{0} t$, where $\Omega_{0}$ is the amplitude coupling constant. The normalized recoil frequency $\omega_{r} \equiv \hbar k_{f}^{2} / m_{a} \Omega_{0} \ll 1$ and the atom-field detuning $\delta \equiv\left(\omega_{f}-\omega_{a}\right) / \Omega_{0}$ are the control parameters. This set possesses an infinite number of the integrals of motion

$$
R_{n} \equiv\left|a_{n}\right|^{2}+\left|b_{n+1}\right|^{2}=\mathrm{const}, \quad \sum_{n=0}^{\infty} R_{n} \leqslant 1,
$$

and conserves the total energy

$$
\begin{aligned}
E \equiv \frac{\omega_{r} p^{2}}{2}-\frac{\delta}{2} \sum_{n=0}^{\infty}\left(\left|a_{n}\right|^{2}-\left|b_{n+1}\right|^{2}\right)- \\
\quad-2 \cos x \sum_{n=0}^{\infty} \sqrt{n+1} \operatorname{Re}\left(a_{n} b_{n+1}^{*}\right) .
\end{aligned}
$$

By introducing new variables

$$
\begin{aligned}
& u_{n} \equiv 2 \operatorname{Re}\left(a_{n} b_{n+1}^{*}\right), \\
& v_{n} \equiv-2 \operatorname{Im}\left(a_{n} b_{n+1}^{*}\right), \\
& z_{n} \equiv\left|a_{n}\right|^{2}-\left|b_{n+1}\right|^{2},
\end{aligned}
$$

we can rewrite the set (3) in the following form:

$$
\begin{gathered}
\dot{x}=\omega_{r} p, \quad \dot{p}=-\sin x \sum_{n} \sqrt{n+1} u_{n}, \\
\dot{u}_{n}=\delta v_{n} \\
\dot{v}_{n}=-\delta u_{n}+2 \sqrt{n+1} z_{n} \cos x \\
\dot{z}_{n}=-2 \sqrt{n+1} v_{n} \cos x
\end{gathered}
$$

with the respective integrals of motion

$$
u_{n}^{2}(\tau)+v_{n}^{2}(\tau)+z_{n}^{2}(\tau)=R_{n}^{2}=\mathrm{const}
$$

and the total energy

$$
E=\frac{\omega_{r} p^{2}}{2}-\frac{\delta}{2} \sum_{n=0}^{\infty} z_{n}-\cos x \sum_{n=0}^{\infty} \sqrt{n+1} u_{n} .
$$

The inverse transformation gives us information about the moduli of the probability amplitudes only

$$
\left|a_{n}\right|^{2}=\frac{R_{n}+z_{n}}{2}, \quad\left|b_{n+1}\right|^{2}=\frac{R_{n}-z_{n}}{2} .
$$

The Hamilton-Schrödinger equations (3) and (8) describe a quantum-classical hybrid with the classical part (the first two equations in the sets), driven by the quantum probability amplitudes, and the quantum one (the other equations in the sets) driven by the envelope of the standing wave. The quantum part is still unitary and therefore cannot suffer from decoherence induced by the classical part. In other words, the total quantum entropy is constant during the evolution. However, the quantum part is a system composed of two quantum subsystems each of which may be characterized by a reduced quantum entropy. The role of the classical motion for the reduced entropy is not trivial and will be clarified in the other sections.

\section{B. Entanglement and fidelity of the atom-field states}

The atomic population inversion $z(\tau)$ is a difference of probabilities to find an atom at the moment of time $\tau$ in the excited and ground states

$$
z \equiv \sum_{n=0}^{\infty}\left|a_{n}\right|^{2}-\sum_{n=0}^{\infty}\left|b_{n}\right|^{2}=\sum_{n=0}^{\infty} z_{n}-\left|b_{0}\right|^{2} .
$$

It is an important characteristic that can be measured in experiments. The entanglement between the internal 
atomic and field degrees of freedom can be characterized by the quantity known as purity

$$
P(\tau) \equiv \operatorname{Tr} \rho_{a}^{2}(\tau),
$$

where $\rho_{a}(\tau)$ is the reduced atomic density matrix

$$
\rho_{a}(\tau) \equiv \sum_{n=0}^{\infty}\langle n|\rho(\tau)| n\rangle
$$

with the total density matrix to be $\rho(\tau) \equiv|\Psi(\tau)\rangle\langle\Psi(\tau)|$. Purity is maximal if an atom is in one of its energetic states $|1\rangle$ or $|2\rangle$, i. e. $P_{\max }=\operatorname{Tr} \rho_{a}^{2}=\operatorname{Tr} \rho_{a}=1$. Purity is minimal if $\rho_{a}=I / 2$, i. e. $P_{\min }=1 / 2$, where $I$ is the identity matrix. In terms of the probability amplitudes, it is given by

$$
\begin{gathered}
P=A^{2}+B^{2}+2|C|^{2} \\
A \equiv \sum_{n=0}^{\infty}\left|a_{n}\right|^{2}, \quad B \equiv \sum_{n=0}^{\infty}\left|b_{n}\right|^{2}, \quad C \equiv \sum_{n=0}^{\infty} a_{n} b_{n}^{*} .
\end{gathered}
$$

As similar standard measures of quantum "disorder", we will use the reduced linear entropy

$$
S_{L} \equiv 1-\operatorname{Tr} \rho_{a}^{2}=1-P
$$

and the reduced von Neumann entropy

$$
\begin{gathered}
S_{N} \equiv-\operatorname{Tr}\left(\rho_{a} \ln \rho_{a}\right)=-\lambda_{1} \ln \lambda_{1}-\lambda_{2} \ln \lambda_{2}, \\
\lambda_{1,2}=\frac{1}{2} \pm \sqrt{\frac{1}{4}+|C|^{2}-A B},
\end{gathered}
$$

where $\lambda_{1,2}$ are eigenvalues of the matrix $\rho_{a}$.

To quantify instability of the Jaynes-Cummings quantum evolution we use the fidelity $f(\tau)$ which is the overlap of two states $\left|\Psi_{1}(\tau)\right\rangle$ and $\left|\Psi_{2}(\tau)\right\rangle$, identical at $\tau=0$, that evolve under two Hamiltonians (1) with slightly different values of one of the system's parameters $\kappa$. In terms of the probability amplitudes it has the form

$$
\begin{aligned}
f(\tau, \kappa, \Delta \kappa) \equiv|\langle\Psi(\tau, \kappa) \mid \Psi(\tau, \kappa+\Delta \kappa)\rangle|^{2}= \\
=\mid \sum_{n=0}^{\infty}\left(a_{n}^{*}(\tau, \kappa) a_{n}(\tau, \kappa+\Delta \kappa)+\right. \\
\left.\quad+b_{n}^{*}(\tau, \kappa) b_{n}(\tau, \kappa+\Delta \kappa)\right)\left.\right|^{2},
\end{aligned}
$$

where $\Delta \kappa$ is a small variation of the parameter $\kappa$. In difference from the reduced quantum entropies introduced above, fidelity is a characteristic of the whole quantum system, not of its part.

\section{Analytical solutions with arbitrary field states}

The Jaynes-Cummings dynamics of a single two-level atom at rest, interacting with a single mode of the quantized field, has been studied in detail by many authors (for a review, see [23]). We are interested here how the dynamical coupling between the Jaynes-Cummings and classical degrees of freedom changes both the quantum and classical dynamics.

The Hamilton-Schrödinger equations (3) are, in general, non-integrable. The type of the center-of-mass motion depends strongly on the values of the detuning $\delta$. In the limit of zero detuning and with initially excited or deexcited atoms, the optical potential disappears, and atoms moves with a constant velocity $\dot{x}=\omega_{r} p_{0}$. The quantum evolution is periodic with the period $\pi / \omega_{r} p_{0}$, and exact solutions for purity, von Neumann entropy, and other quantum characteristics can be found in the explicit form. With arbitrary initial field and atomic states, the variables $u_{n}$ are constants, $u_{n}=u_{n}(0)$, for each $n$. The Hamilton equations for the translational degree of freedom are closed, and their solution can be easily found in terms of the Jacobi elliptic functions [1]]. For each $n$, we get the following exact solution:

$$
\begin{aligned}
& z_{n}(\delta=0)= \\
& =\mp \sqrt{R_{n}^{2}-u_{n}^{2}(0)} \sin \left(2 \sqrt{n+1} \int \cos x(\tau) d \tau+\varphi_{n}\right), \\
& \varphi_{n} \equiv \mp \arcsin \left(z_{n}(0) / \sqrt{R_{n}^{2}-u_{n}^{2}(0)}\right)
\end{aligned}
$$

that describes a frequency modulated signal. Exact solutions for the amplitudes $a_{n}$ and $b_{n}$ are the following:

$$
\begin{aligned}
& a_{n}(\delta=0)=a_{n}(0) \cos \left(\sqrt{n+1} \int \cos x(\tau) d \tau\right)+ \\
& +i b_{n+1}(0) \sin \left(\sqrt{n+1} \int \cos x(\tau) d \tau\right), \quad(20) \\
& b_{n+1}(\delta=0)=b_{n+1}(0) \cos \left(\sqrt{n+1} \int \cos x(\tau) d \tau\right)+ \\
& +i a_{n}(0) \sin \left(\sqrt{n+1} \int \cos x(\tau) d \tau\right),
\end{aligned}
$$

where $a_{n}(0)$ and $b_{n+1}(0)$ are the initial complex values of $a_{n}$ and $b_{n+1}$. In the Raman-Nath limit, $p \simeq p_{0}=$ const, the solution (19) is simplified

$$
\begin{aligned}
& z_{n}(\delta=0) \simeq \\
& \quad \simeq \mp \sqrt{R_{n}^{2}-u_{n}^{2}(0)} \sin \left(\frac{2 \sqrt{n+1}}{\omega_{r} p_{0}} \sin \omega_{r} p_{0} \tau+\varphi_{n}\right) .
\end{aligned}
$$

With the detuning being large, $|\delta| \gg 1$, the optical potential is shallow, atom moves with almost a constant velocity, $\simeq \omega_{r} p_{0}$, slightly modulated by the standing wave, and its inversion oscillates with a small depth (excepting for the case of the so-called Doppler-Rabi resonance with maximal Rabi oscillations that occur at the condition $\left.|\delta| \simeq \omega_{r}\left|p_{0}\right|[24]\right)$. If the atomic kinetic energy $\omega_{r} p^{2} / 2$ is not enough to overcome barriers of the optical potential, 
the atomic center of mass oscillates nonlinearly in one of the potential wells.

With very fast atoms, $\omega_{r}\left|p_{0}\right| \gg \max (|\delta|, 2 \sqrt{n+1})$, or large detunings, $|\delta| \gg \max \left(\omega_{r}\left|p_{0}\right|, 2 \sqrt{n+1}\right)$, one can get the approximate amplitude-modulated solutions for $z_{n}$

$$
\begin{gathered}
z_{n} \simeq z_{n}(0)-\frac{2 \sqrt{\left(R_{n}^{2}-z_{n}^{2}(0)\right)(n+1)}}{\omega_{r} p_{0}} \cos \left(\delta \tau+\phi_{n}\right) \sin x, \\
\omega_{r}\left|p_{0}\right| \gg \max (\delta, 2 \sqrt{n+1}) ; \\
z_{n} \simeq z_{n}(0)+\frac{2 \sqrt{n+1} u_{n}(0)}{\delta}- \\
-\frac{2 \sqrt{\left(R_{n}^{2}-z_{n}^{2}(0)\right)(n+1)}}{\delta} \cos x \sin \left(\delta \tau+\phi_{n}\right), \\
\delta \gg \max \left(\omega_{r}\left|p_{0}\right|, 2 \sqrt{n+1}\right),
\end{gathered}
$$

where $\phi_{n} \equiv \arcsin \left(u_{n}(0) / \sqrt{R_{n}^{2}-z_{n}^{2}(0)}\right)$. In both the cases, we can find the respective approximate solutions for fidelity, i.e. overlap (18) of two initially identical quantum states, evolving under Hamiltonians with slightly different values of the detuning $\Delta \delta$

$$
f(\tau, \delta, \Delta \delta) \simeq A^{2}(0)+B^{2}(0)+2 A(0) B(0) \cos \Delta \delta \tau,
$$

where $A(0), B(0)$ are initial values of $A$ and $B$ defined in (15).

Inspecting the Hamilton-Schrödinger equations (3), we see that the translational motion is described by the equation for a nonlinear oscillator with the frequency modulation which is caused by the Jaynes-Cummings dynamics. It has been proven in Ref. [12] for this type of equations that, as the result of interaction of nonlinear resonances, there arises a stochastic layer in the respective classical phase space. The width of this layer depends on the values of the detuning $\delta$. With the detunings of the order $|\delta| \lesssim 1$, the atomic center of mass can walk in an erratic way inside a cavity with a stationary standing wave. This type of motion may be called a chaotic or random walking, and it is quantified by positive values of the maximal Lyapunov exponent $\lambda$. For a classical standing wave, the Hamiltonian chaos has been studied in detail in Refs. [11, 13, 14] where the dynamical effects of Lévy flights and atomic fractals have been found and described.

\section{ENTANGLEMENT, FIDELITY, AND QUANTUM-CLASSICAL CORRELATION IN A FOCK FIELD}

In this section we describe a quantum-classical correlation for a two-level atom moving in the quantized field prepared initially in a Fock state. If the cavity mode is prepared in the state $|n\rangle$ with an exactly specified number of photons $n$, the infinite-dimensional set of the Hamilton-Schrödinger equations (8) reduces to the following finite-dimensional set which is written in terms of the Bloch-like variables:

$$
\begin{gathered}
\dot{x}=\omega_{r} p, \quad \dot{p}=-\left(\sqrt{n} u_{n-1}+\sqrt{n+1} u_{n}\right) \sin x, \\
\dot{u}_{n-1}=\delta v_{n-1}, \quad \dot{u}_{n}=\delta v_{n}, \\
\dot{v}_{n-1}=-\delta u_{n-1}+2 \sqrt{n} z_{n-1} \cos x, \\
\dot{v}_{n}=-\delta u_{n}+2 \sqrt{n+1} z_{n} \cos x, \\
\dot{z}_{n-1}=-2 \sqrt{n} v_{n-1} \cos x, \quad \dot{z}_{n}=-2 \sqrt{n+1} v_{n} \cos x .
\end{gathered}
$$

In fact, we deal with a two-level quantum system (an atom) entangled with a three-level quantum system (a field state which is a superposition of the three Fock states $|n-1\rangle,|n\rangle$, and $|n+1\rangle)$. The atomic purity (13) can be now expressed in terms of the components of the atomic population inversion $z(\tau)=z_{n-1}(\tau)+z_{n}(\tau)$ and the integrals of motion $R_{n-1}$ and $R_{n}$ (5)

$$
P_{n}=\frac{1}{2}\left[1+\left(z_{n}+z_{n-1}\right)^{2}+\left(R_{n}+z_{n}\right)\left(R_{n-1}-z_{n-1}\right)\right] .
$$

With the atom prepared initially in one of its energetic states, say, in the excited state $|2\rangle$, and the field, prepared in a Fock state $|n\rangle$, we get the simplest possible kind of entanglement between these two two-level quantum systems. The respective purity is extremely simplified

$$
P_{n}=\frac{1}{2}\left(1+z_{n}^{2}\right)
$$

We can now analyze in detail the correlations between the quantum entanglement and the center-of-mass motion. At exact resonance $\delta=0$, atoms, initially prepared in one of the energetic states, fly through a cavity with a constant velocity $p_{0}$, and using (19) we get exact solution for the purity

$$
P_{n}(\delta=0)=\frac{1}{2}+\frac{1}{2} \cos ^{2}\left(\frac{2 \sqrt{n+1}}{\omega_{r} p_{0}} \sin \omega_{r} p_{0} \tau\right),
$$

oscillating periodically between $1 / 2$ and 1 .

In the other limiting cases of fast atoms, $\omega_{r}\left|p_{0}\right| \gg \max (|\delta|, 2 \sqrt{n+1})$, and large detunings, $|\delta| \gg \max \left(\omega_{r}\left|p_{0}\right|, 2 \sqrt{n+1}\right)$, the atomic center-of-mass motion is regular (nonlinear oscillations in a well of the optical potential or a ballistic flight with almost a constant velocity $\omega_{r} p_{0}$ slightly modulated by the standing wave), the atomic inversion oscillates with a small depth, and the atomic purity is $P_{n} \simeq 1$. But oscillations of the purity can be large with periodically maximal entanglement between the atom and the Fock field, if the condition of the Doppler-Rabi resonance, $\omega_{r}|p| \simeq|\delta|$, is fulfilled. When the atom comes into the resonance with one of the running waves, composing a standing wave, the Rabi oscillations become maximal. Using the solution for the atomic inversion found in [13], it is easy to get the respective atomic purity

$$
\begin{aligned}
& P_{n}\left(|\delta| \simeq \omega_{r}|p|\right) \simeq \\
& \simeq \frac{1}{2}+\frac{1}{2}\left(\frac{\left(|\delta|-\omega_{r}\left|p_{0}\right|\right)^{2}}{\Omega_{n}^{2}}+\frac{\sqrt{n+1}}{\Omega_{n}^{2}} \cos \Omega_{n} \tau\right)^{2},
\end{aligned}
$$


oscillating periodically with the Rabi frequency $\Omega_{n} \equiv$ $\sqrt{\left(|\delta|-\omega_{r}\left|p_{0}\right|\right)^{2}+n+1}$ and the maximal amplitude at $|\delta|=\omega_{r}\left|p_{0}\right|$.

Chaotic walking of the atomic center of mass in a standing wave which is initially prepared in a Fock state, is expected to occur in the detuning range $|\delta| \lesssim 1$ for the chosen values of the recoil frequency $\omega_{r}=0.001$ and the number of photons $n=10$. In Fig. 1 we plot the evolution of the atomic purity $P_{n}(\tau)$ (the left panel) and the respective power spectra (the right panel) in different situations: (a) exact resonance $\delta=0$ and $p_{0}=25$, (b) a ballistic flight with large detuning $\delta=32$ and $p_{0}=25$, (c) the Doppler-Rabi resonance with $\delta=32$ and $p_{0}=32000$, and (d) a chaotic walking with $\delta=0.4$ and $p_{0}=25$. At exact resonance, the oscillations of purity are frequency modulated but strictly periodic with the period $\pi / \omega_{r} p_{0} \simeq 125.6$. The spectrum consist of a variety of resolved peaks in the frequency range from $\sim 0.01$ to 2.2 in units of the vacuum Rabi frequency $\Omega_{0} / 2 \pi$ (in the right panel of Fig. 10 we show only the low-frequency and highfrequency parts of the whole spectrum). Off-resonant oscillations of purity is an amplitude-modulated signal the spectrum of which contains mainly high frequencies (see Fig. 10 where we cut off for convenience the part of spectrum from 0.05 to 5 that does not contain any pronounced peaks). The Doppler-Rabi oscillations and its spectrum (Fig. 1. contain the main harmonic with $\omega / 2 \pi \simeq 1$ and a few high frequencies. Purity oscillations with a randomly walking atom look like chaotic ones with a broadened spectrum in the frequency range from 0 to 2.2 (Fig. 11).

The chaotic centre-of-mass walking in the quantized field has fractal properties similar to those that have been found with atoms in a classical field 13. Placing atoms at the point $x=0$ with the same initial conditions and parameters but with different values of initial momenta $p_{0}$, we compute the time $T\left(p_{0}\right)$, the atom with a given value $p_{0}$ needs to reach one of the nodes of the standing wave at $x=-\pi / 2$ and $x=3 \pi / 2$, and the number of times $m$ when it changes its direction of motion. The scattering function $T\left(p_{0}\right)$ is found to have a self-similar structure with singularities on a Cantor-like set of initial values of momenta $p_{0}$. In Fig. 2 2 we demonstrate the mechanism of generating this set at $\delta=0.4$ in the initial Fock field with $n=10$ and initially excited atoms. The exit-time function demonstrates an intermittency of smooth curves and complicated structures that cannot be resolved in principle, no matter how large the magnification factor. Fig. 20 shows magnification of the function for the small interval $45.9 \leqslant p_{0} \leqslant 46.9$. Further magnifications reveal a self-similar structure.

There are two sets of atomic trajectories with $T \rightarrow \infty$, the countable one consisting of separatrix-like trajectories, corresponding to the ends of the intervals in Fig. 2 and the uncountable one consisting of trajectories with $m=\infty$. The chaotic motion can be, in principle, verified in experiments on 1D-scattering of atoms at the standing wave. Fig. [3 demonstrates a sensitive dependence of the atomic positions on $p_{0}$ at a fixed time moment. A smooth segment of this function in the range $\left|p_{0}\right| \lesssim 20$ should be attributed to atomic center-of-mass oscillations in the first well of the optical potential since these values of $p_{0}$ are not enough to overcome the respective potential barrier. When $p_{0}$ exceeds a critical value, atoms leave the well, and it is practically impossible to predict even the sign of the atomic position. The socalled predictability horizon can be estimated as follows: $\tau_{p} \simeq \lambda^{-1} \ln \left(\Delta x / \Delta x_{0}\right)$, where $\Delta x$ is the confidence interval and $\Delta x_{0}$ the inaccuracy in preparing initial atomic positions. In order to demonstrate the quantum-classical correspondence in the chaotic regime we compute the dependence of the output values of the atomic population inversion $z_{\text {out }}$ at a fixed moment on its initial values $z_{\text {in }}$ with the other conditions and parameters to be the same. Fig. [ shows that the predictability horizon of the quantum atomic degree of freedom can be estimated to be $\tau \simeq 200$.

The oscillations of purity of a chaotically moving atom and its spectrum look like irregular ones (Fig. 11). To quantify the irregularity we compute the root mean square variance of purity $\sigma_{P} \equiv \sqrt{\left\langle P^{2}\right\rangle-\langle P\rangle^{2}}$ on a large time scale (with $\langle P\rangle$ being a purity value averaged on this scale) in the range of the detuning $|\delta| \leq 2$ and compare the result with the dependence $\lambda(\delta)$ in the same range, where the maximal Lyapunov exponent has been computed with the Fock system (25) with the atom prepared at $\tau=0$ in the superposition state $(|1\rangle+|2\rangle) / \sqrt{2}$ $(z(0)=0)$ and initial conditions $x_{0}=0$ and $p_{0}=25$. Fig. [ 5 demonstrates that irregular oscillations of $\sigma_{P}$ occur on the same interval of the detuning $\delta$, where $\lambda>0$. Since similar correlations has been found with different initial atomic and Fock states not only for the linear entropy but for the von Neumann entropy $S_{N}$ as well, we may conclude that the correlation between the quantum entanglement and the classical motion has been established. The role of the translational atomic motion for purity is transparent with the Fock field when $P$ is expressed in terms of $z_{n}$ which are variables of two oscillators coupled to the classical $x-p$ oscillator (see Eqs. (25)). When the latter one oscillates regularly, the first ones do the same. When an atom walks randomly in a cavity, it immediately causes chaotic oscillations in $z_{n}$ variables and therefore in purity.

To quantify instability of quantum evolution of the atom-field system we compute the decay of the fidelity $f(\tau)$ which is the overlap (18) of two states $\left|\Psi_{1}(\tau)\right\rangle$ and $\left|\Psi_{2}(\tau)\right\rangle$, identical at $\tau=0$, that evolve under two Hamiltonians (11) with the slightly different detunings $\delta$ and $\delta^{\prime} \equiv \delta+\Delta \delta$. Time evolution of fidelity with the Fock initial state $|n=10\rangle$ has been computed with different values of the detuning and the other conditions to be the same as specified above. For those values of $\delta$, when the center-of-mass motion is chaotic and the maximal Lyapunov exponent is positive, we have found that fidelity 

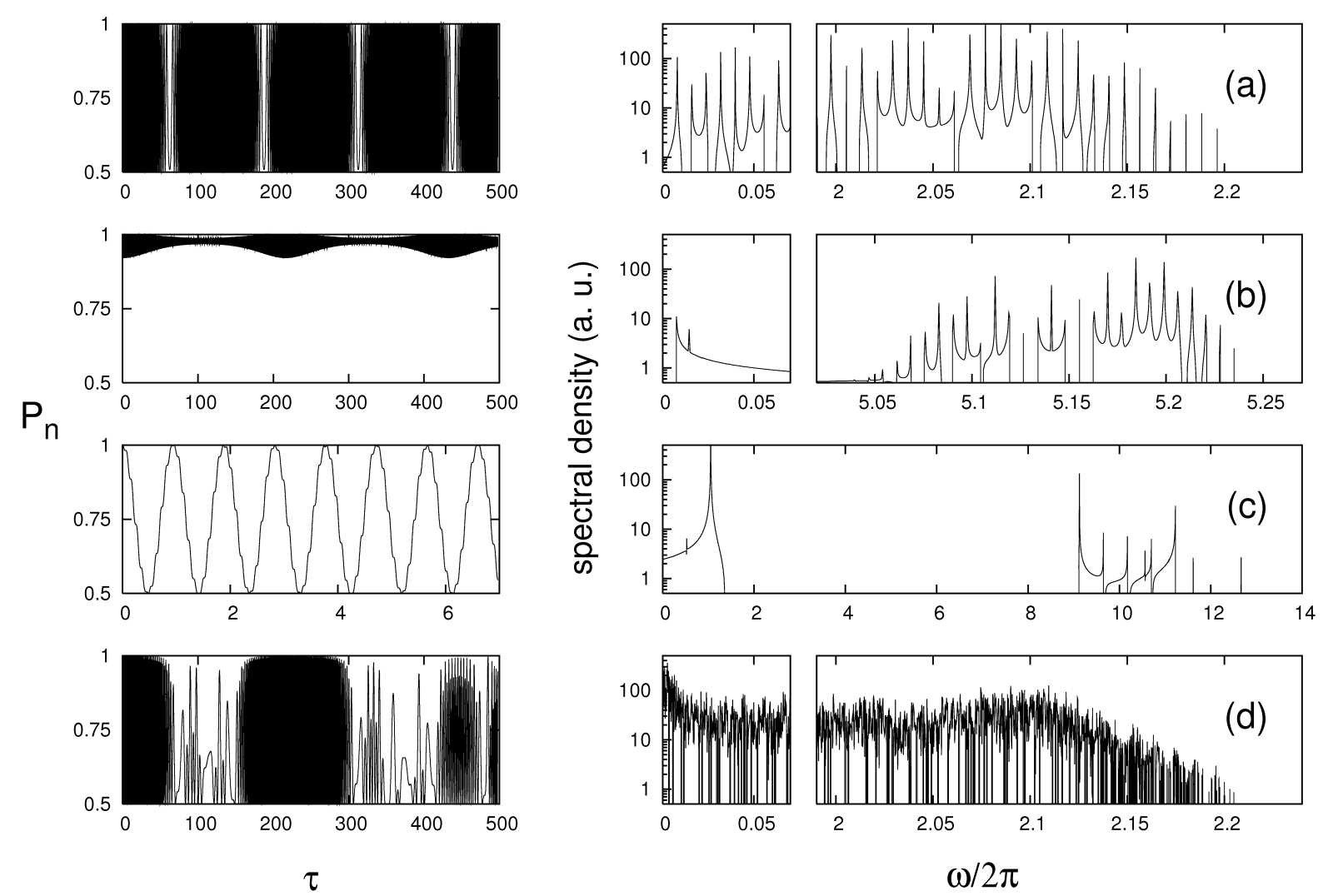

FIG. 1: Left panel: time evolution ( $\tau$ is in units of $\Omega_{0}^{-1}$ ) of the atomic purity $P_{n}$ with an atom initially prepared in the excited state and the field initially in a Fock state with $n=10$. (a) Exact resonance, $\delta=0$ and $p_{0}=25$, (b) a ballistic atom, $\delta=32$ and $p_{0}=25$, (c) the Doppler-Rabi resonance with $\delta=32$ and $p_{0}=32000$, (d) chaotic walking with $\delta=0.4$ and $p_{0}=25$. Right panel: the respective power spectra, with the frequency $\omega$ in units of $\Omega_{0}$.
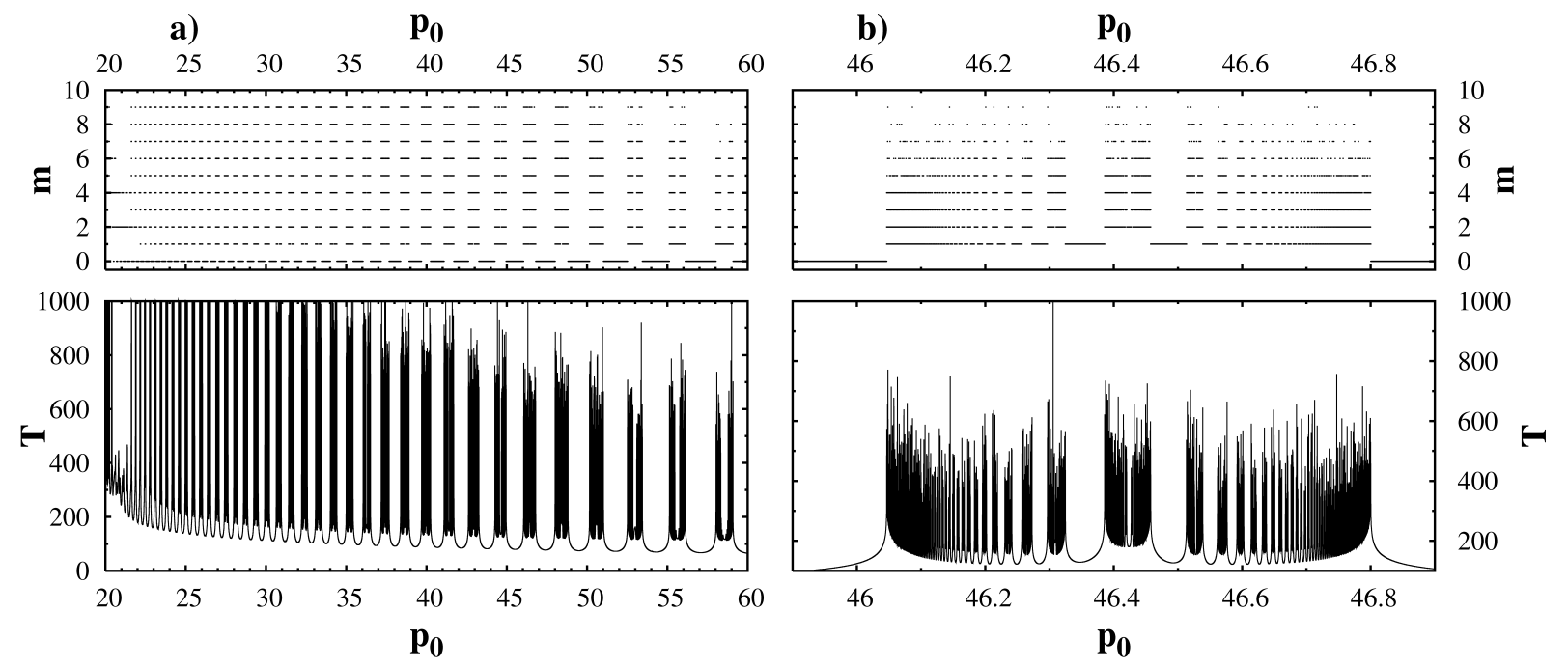

FIG. 2: Fock atomic fractal (a) and a zoom of one of its fragment (b) at $n=10, \delta=0.4$, and $z(0)=1$. The upper panels: how many times $m$ an atom with a given initial momentum $p_{0}$ (in units of $\hbar k_{f}$ ) changes its direction of motion before leaving a cavity. The lower panels: the time $T$ (in units of $\Omega_{0}^{-1}$ ) atoms with given values of the initial momenta $p_{0}$ spend in the cavity before leaving. 


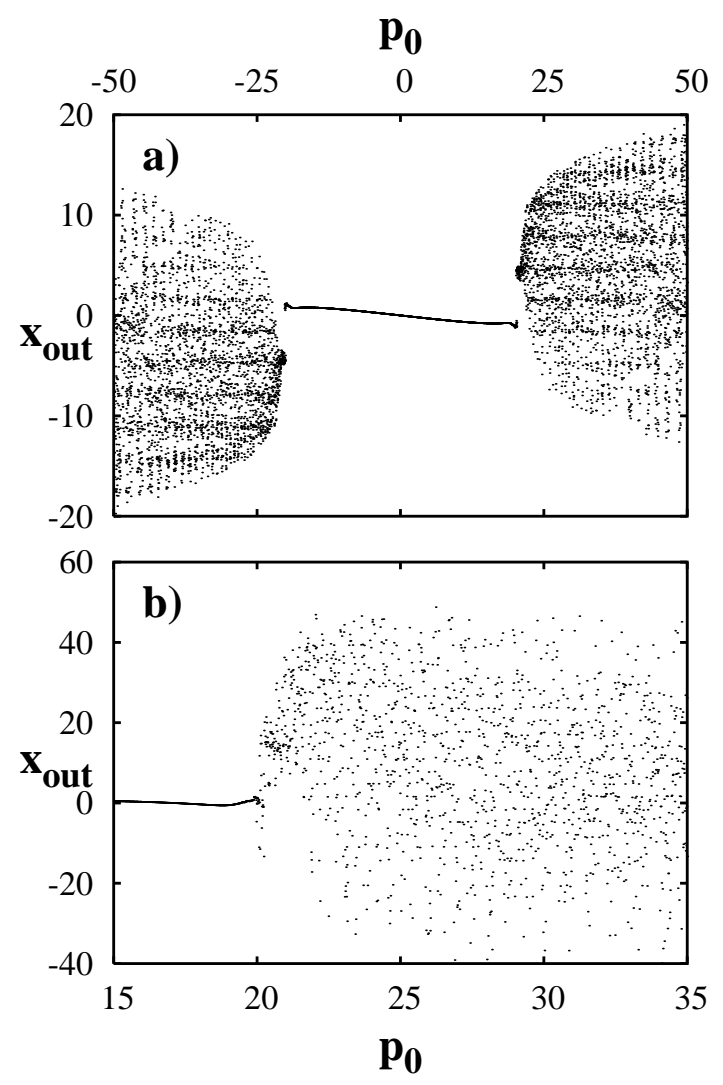

FIG. 3: Sensitive dependence of the atomic position $x_{\text {out }}$ (in units of $k_{f}^{-1}$ ) on the initial momentum $p_{0}$ at (a) $\tau=300$ and (b) $\tau=1000$. All the other conditions are the same as in Fig. 2

rapidly decays to its minimal value $f=0$ with the rate given approximately by the respective value of $\lambda$, and afterwards $f$ oscillates in an irregular manner. For the values of $\delta$, where the center-of-mass motion is regular and $\lambda \simeq 0$, fidelity evolves much more regularly. In Fig. 6 we show for convenience the evolution of the quantity $\log _{10}(1-f)$ in the chaotic (solid line, $\delta=0.4$ ) and regular regimes of the center-of-mass motion (dotted line, $\delta=0.8$ ) with $\Delta \delta=10^{-4}$. The results obtained do not depend qualitatively on the values of differences in the detuning $\Delta \delta$.

The time evolution of fidelity can be explained as follows. With an initially excited atom, the fidelity is

$$
\begin{aligned}
f(\tau, \delta, \Delta \delta)=\mid a_{n}^{*}(\tau, \delta) a_{n}(\tau, \delta+\Delta \delta)+ & \\
& \quad+\left.b_{n+1}^{*}(\tau, \delta) b_{n+1}(\tau, \delta+\Delta \delta)\right|^{2}
\end{aligned}
$$

where $a_{n}$ and $b_{n+1}$ are variables of a quantum oscillator coupled to the classical $x-p$ oscillator. At $\delta=0.4$ and with any other values of $\delta$ when the center-of-mass motion is chaotic, all the oscillator variables are sensitive dependent not only to small variations of initial conditions but to the control parameters as well. However, the probability amplitudes and fidelity are restrictive quantities.
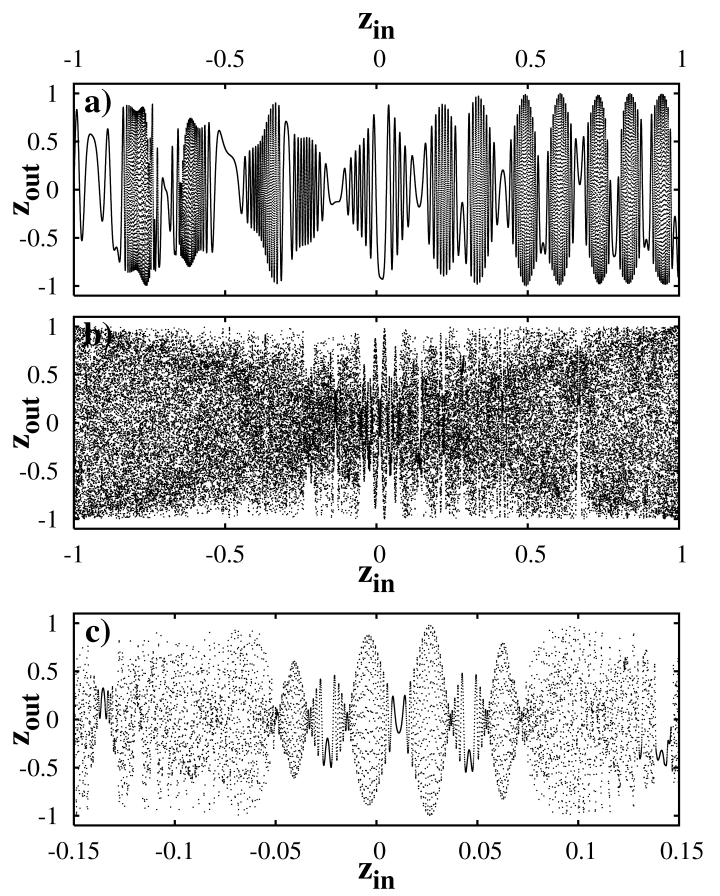

FIG. 4: Sensitive dependence of the output values of the atomic population inversion $z_{\text {out }}$ on its initial values $z_{\text {in }}$. (a) $\tau=100$, (b) $\tau=200$, and (c) zoom of a small interval of $z_{\text {in }}$ around $z_{\text {in }}=0$ at $\tau=200$.

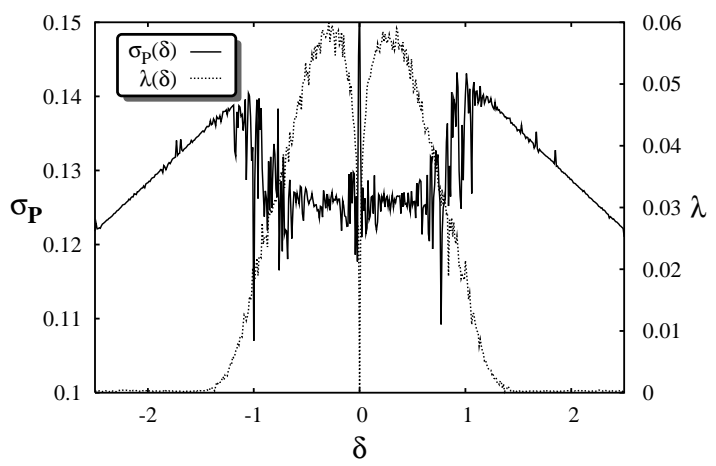

FIG. 5: Quantum-classical correlation between the dependencies of the variance of the purity $\sigma_{P}$ and the maximal Lyapunov exponent $\lambda$ (in units of $\Omega_{0}$ ) on the atom-field detuning $\delta$ (in units of $\Omega_{0}$ ). Initial Fock field with $n=10$ and an atom prepared initially in the superposition state $(|1\rangle+|2\rangle) / \sqrt{2}$.

Because of an initial exponential divergence of the quantities $a_{n}(\tau, \delta), a_{n}(\tau, \delta+\Delta \delta), b_{n+1}(\tau, \delta)$, and $b_{n+1}(\tau, \delta+\Delta \delta)$, fidelity rapidly decays up to its minimal value $f=0$ with the rate approximately given by the maximal Lyapunov exponent of the Hamilton-Schrödinger equations for a chaotically moving atom (solid line in Fig. 6). After that, it oscillates irregularly in a large range. At $\delta=0.8$ and at any other value of the detuning when atoms move regularly in a cavity, fidelity $f$ decays much more slowly as compared to the chaotic case and oscillates smoothly (dotted line in Fig. [6). 


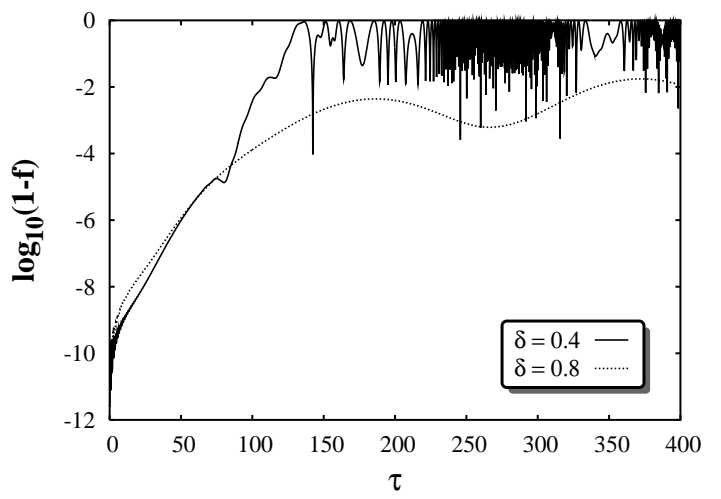

FIG. 6: Time evolution of quantum fidelity (logarithmic scale) in the chaotic (solid line at $\delta=0.4$ ) and regular (dotted line at $\delta=0.8$ ) regimes of the center-of-mass motion in a Fock field with $n=10$.

\section{ENTANGLEMENT, FIDELITY, AND QUANTUM-CLASSICAL CORRELATION IN A COHERENT FIELD}

In this section we consider the quantized field in a cavity to be supposed initially in a coherent state

$$
|\alpha\rangle=e^{-|\alpha|^{2} / 2} \sum_{n=0}^{\infty} \frac{\alpha^{n}}{\sqrt{n !}}|n\rangle,
$$

where $|\alpha|^{2}=\langle n\rangle$ is an average number of photons in the state (31). The equations of motion (3) and (8) are now infinite dimensional, and their analytic solutions have been derived in a few limiting cases in Sec. IIC

Because of an infinite number of incommensurate Rabi frequencies, the population inversion of an atom in an initially coherent field oscillates in a complicated way. In the Raman-Nath limit, the inversion $z(\tau)$, the atomic purity $P(\tau)$, and the entropies $S_{L}$ and $S_{N}$ are periodic functions with the period $\pi / \omega_{r} p_{0}$ given by the time of the atomic flight between two neighboring nodes of the standing wave. Out of resonance, the center-of-mass motion is regular at $|\delta| \gtrsim 1$ and chaotic in approximately the same range, $|\delta| \lesssim 1$, as in the Fock field.

In numerical simulation we truncate the set (3) at a finite $n=100$ which is sufficient for all our purposes. Fig. [7 demonstrates the correlation between $\delta$-dependencies of the variance of the atomic purity $\sigma_{P}$ and of the maximal Lyapunov exponent $\lambda$ with the atom prepared initially in the energetic state $|2\rangle$ and the field at $\tau=0$ in the coherent state (31) with $\bar{n}=10$ and the other conditions and parameters to be the same as for the Fock field. Comparing Figs. 5 and 7 we see that the quantum-classical correlations in the coherent and Fock fields are similar despite of the fact that purity in a coherent field even in the limiting cases oscillates in a much more complicated way as compared to purity in a Fock field.

In the regime of chaotic walking, atoms in a coherent field demonstrate fractal scattering (Fig. 8 a ) and sensitive dependencies on the initial states both in the classical

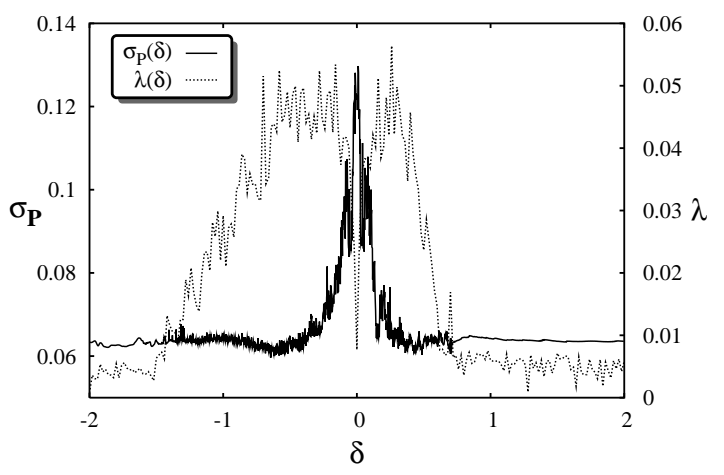

FIG. 7: Quantum-classical correlation between the dependencies of the variance of quantum purity $\sigma_{P}$ and the maximal Lyapunov exponent $\lambda$ (in units of $\Omega_{0}$ ) on the atom-field detuning $\delta$ (in units of $\Omega_{0}$ ). Initial coherent field with $\bar{n}=10$ and an atom prepared initially in the excited state. The other conditions are the same as in Fig. 5
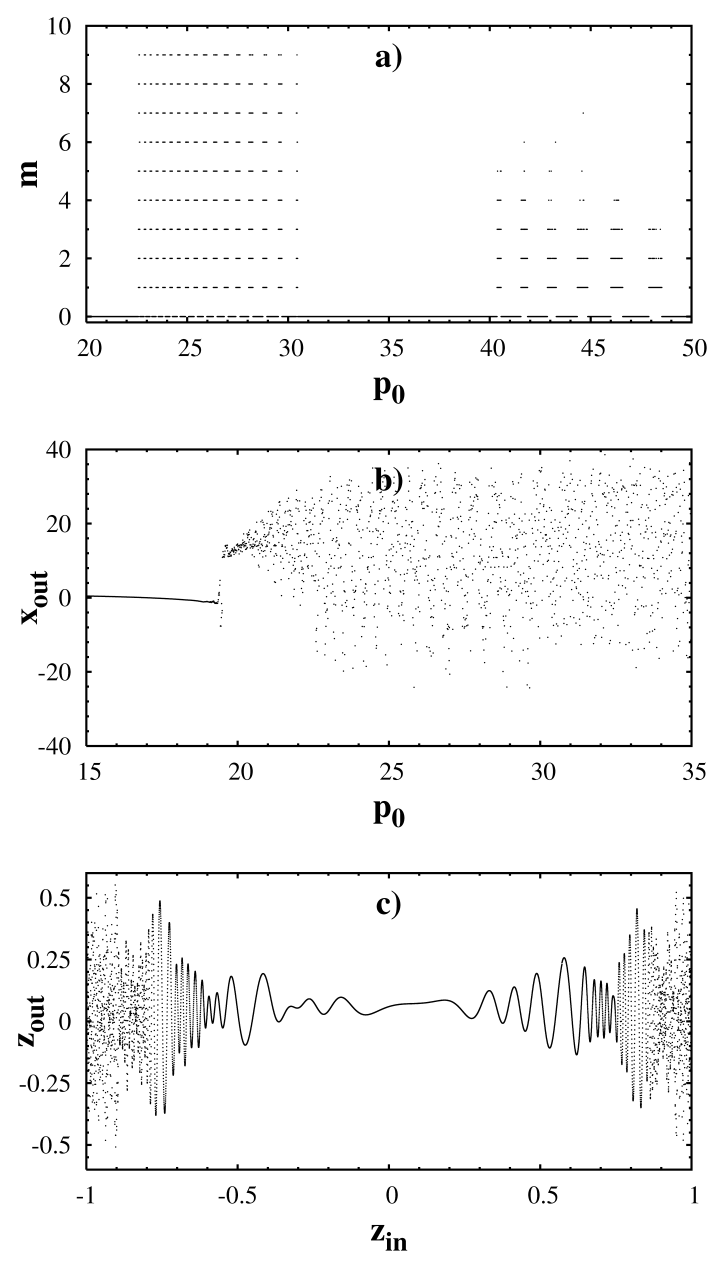

FIG. 8: (a) Fractal set of the initial momenta $p_{0}$ (in units of $\hbar k_{f}$ ) of atoms which leave a cavity with initially coherent field after $m$ turns. (b) Sensitive dependence of the atomic position $x_{\text {out }}$ (in units $k_{f}^{-1}$ ) on the initial momentum $p_{0}$. (c) Sensitive dependence of the output values of the atomic population inversion $z_{\text {out }}$ on its initial values $z_{\text {in }}$. Control parameters $\delta=0.4, \bar{n}=10$, and $\omega_{r}=0.001$. 


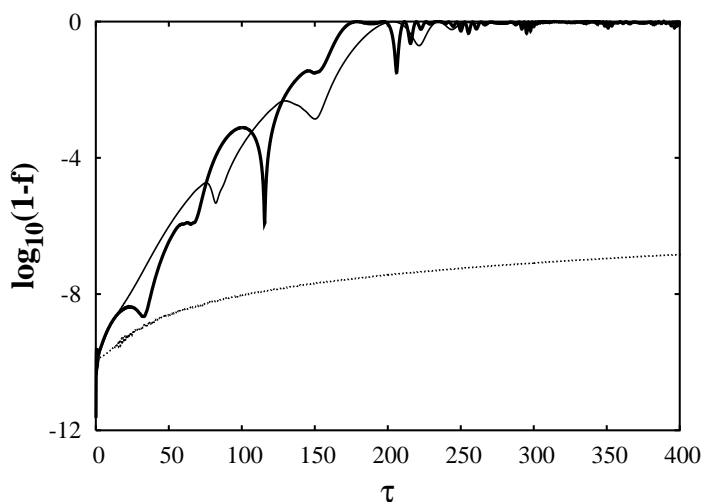

FIG. 9: Time evolution of quantum fidelity (logarithmic scale) in the chaotic (thick and thin lines) and regular (dotted line) regimes of the center-of-mass motion in a coherent field with $\bar{n}=10$.

(Fig. 8b) and quantum (Fig. 88) degrees of freedom. The strength of chaos, measured by the value of $\lambda$, is the same for both the initial field states $|n\rangle$ and $|\alpha\rangle$ (see Figs. 5 and 17). The function $z_{\text {out }}\left(z_{\text {in }}\right)$ is regular in a wider range of $z_{\text {in }}$ with atoms in a coherent field (Fig. 8k) than with atoms in a Fock field (Fig. 4) simply because of choosing a specific value of the phase of the coherent state $|\alpha\rangle$.

We have found (see Fig. 8) that with the initial momentum $p_{0}=25$ and $\delta=0.4$ the type of atomic motion depends strongly on the initial atomic inversion $z(0)$. If an atom is prepared initially in one of its energetic states, i. e. $z(0)= \pm 1$, its classical and quantum dynamics are unstable, whereas they are stable with $z(0)=0$ under the same other conditions. In Fig. 9 we show the evolution of the quantity $\log _{10}(1-f)$ in the regimes of chaotic walking (thick and thin lines, $z(0)= \pm 1$ ) and regular motion (dotted line, $z(0)=0$ ) with $\Delta \delta=10^{-4}$. In the chaotic regime the fidelity initially decays exponentially with the rate $\lambda \simeq 0.04$ to be equal to the maximal Lyapunov exponent computed with the set (3). This result does not depend on the values of differences in the detuning $\Delta \delta$. The fidelity practically does not decay in the regular regime at $z(0)=0$, and the respective maximal Lyapunov exponent was computed to be zero.

With randomly walking atoms, initial decay of fidelity is practically the same both in the Fock (Fig. 6) and coherent (Fig. 9) initial field states and is quantified by the respective Lyapunov exponents. After reaching zero value, fidelity demonstrates erratical oscillations in both the cases. These oscillations are more pronounced with the Fock field because entanglement there occurs between a few quantum states, whereas entanglement with a coherent field implies, in principle, an infinite number of states. In any case, behavior of fidelity strongly differs with regularly and randomly walking atoms.

\section{CONCLUSION}

We have found the quantum-classical correspondence in the basic model in cavity QED by proving that entanglement between electronic and photonic degrees of freedom and fidelity of the Jaynes-Cummings dynamics correlate with the center-of-mass motion of a two-level atom in a quantized standing-wave cavity field. It has been shown analytically and numerically both with initial Fock and coherent field states that quantum entropy, purity, and fidelity of regularly moving atoms evolve in a regular way, whereas the respective quantum evolution is unstable with atoms moving chaotically in a periodic standing wave. Instability of the quantum evolution has been shown to be quantified by the respective classical maximal Lyapunov exponent for different initial electronic and field states and with different values of the main control parameter, the atom-field detuning $\delta$. We emphasize that this quantum instability and irreversibility is caused by internal dynamical chaos and takes place without any external environment.

We have done some numerical experiments and found various manifestations of the quantum-classical correspondence, including dynamical chaos and fractals, which can be, in principle, observed in real experiments with atoms and photons in high-finesse cavities. In this connection, we should mention first of all pioneering experiments of Kimble's and Rempe's groups 25] on real-time position control of a single atom in a high-finesse microcavity where a photon may be periodically absorbed by the atom and re-emitted into the cavity many times before being lost outside the cavity. In the strong-coupling regime, when the coherent coupling between a single atom and intracavity field dominates atomic spontaneous emission and intracavity-field decay, the center-of-mass position within the cavity mode can be monitored in real time with high spatial and temporal evolution by detecting the light transmitted by the cavity. These achievements open up new possibilities in the control and continuous measurement of the internal and external dynamical variables of atoms.

\section{ACKNOWLEDGMENTS}

The work was supported by the Program "Mathematical methods in nonlinear dynamics" of the Russian Academy of Sciences and by the Far Eastern Division of the Russian Academy of Sciences.
[1] E.T. Jaynes and E.W. Cummings, Proc. IEEE 51, 89 (1963).
[2] R.M. Dicke, Phys. Rev. 93, 493 (1954).

[3] P.I. Belobrov, G.M. Zaslavskii, and G.Kh. Tartakovskii, 
Zh. Eksp. Teor. Fiz. 71, 1799 (1976) [Sov. Phys. JETP 44, 945 (1976)]

[4] J. R. Ackerhalt, P. W. Milonni, and M. -L. Shih, Phys. Rep. 128, 205 (1985).

[5] K. Furuya, M.C. Nemes, and G.Q. Pellegrino, Phys. Rev. Lett. 80, 5524 (1998).

[6] Xi-Wen Hou and Bambi Hu, Phys. Rev. A 69, 042110 (2004).

[7] P.A. Miller and S. Sarkar, Phys. Rev. E 60, 1542 (1999).

[8] Q. Xie and W. Hai, Eur. Phys. J. D 33, 265 (2005).

[9] S.V. Prants, J. Russ. Laser Res. 18, 69 (1997).

[10] L. Braun, W.T. Strunz, and J. Briggs, Phys. Rev. A 70, 033814 (2004).

[11] S. V. Prants and V. Yu. Sirotkin, Phys. Rev. A 64, 033412 (2001).

[12] S.V. Prants, Pis'ma Zh. Éksp. Teor. Fiz. 75, 71 (2002) [JETP Letters 75, 63 (2002)].

[13] V.Yu. Argonov and S.V. Prants, Zh. Eksp. Teor. Fiz. 123, 946 (2003) [JETP 96, 832 (2003)].

[14] S. V. Prants, M. Edelman, and G. M. Zaslavsky, Phys. Rev. E 66, 046222 (2002).

[15] B.V. Chirikov, Chaos 1, 95 (1991).

[16] A. Peres, Phys. Rev. A 30, 1610 (1984).
[17] R. Schack and C.M. Caves, Phys. Rev. E 53, 3257 (1996).

[18] R.A. Jalabert and H.M. Pastawski, Phys. Rew. Lett. 86, 2490 (2001).

[19] Ph. Jacquod, P.G. Silvestrov, and C.W.J. Beenakker, Phys. Rev. E 64, 055203(R) (2001).

[20] G. Benenti and G. Casati, Phys. Rev. E 65, 066205 (2002).

[21] T. Prosen, T.H. Seligman, and M. Znidaric, Phys. Rev. A 67, 042112 (2003).

[22] S.V. Prants and M.Yu. Uleysky, Phys. Lett. A 309, 357 (2003).

[23] B.W. Shore and P.L. Knight, J. Mod. Opt. 40, 1195 (1993).

[24] M.Yu. Uleysky, L.E. Kon'kov, and S.V. Prants, Comm. Nonlin. Sci. Numer. Simul. 8, 329 (2003).

[25] C.J. Hood, T.W. Lynn, A.C. Doherty, A.S. Parkins, and H.J. Kimble, Science 2871147 (2000); P.W.H. Pinkse, T. Fischer, P. Maunz, and G. Rempe, Nature, 404365 (2000); P. Horak, H. Ritsh, T. Fischer, et al., Phys. Rev. Lett. 88, 043601 (2002); T.W. Lynn. K. Birnbaum, and H.J. Kimble. E-print quant-ph/0507064 (2005). S. Husmann, et al. E-print quant-ph/0506088 (2005). 